\title{
An Existence Theorem for Optimal Control Systems with State Variable in $C$, and Stochastic Control Problems ${ }^{\mathrm{i}}$
}

\author{
RICHARD F. BAUM ${ }^{2}$ \\ Communicated by L. Cesari
}

\begin{abstract}
We consider an existence theorem for control systems whose state variables for every $t$ are in $C$, the set of continuous functions varying over a given set $I$. The dependence of the state variables upon $a \in I$ is induced by their dependence upon the initial state and the state equation governing the system. In contrast, the control $u=u(t)$ is taken as a measurable function of $t$ alone. The usual space constraints and boundary conditions are also allowed to vary over $a \in I$, and the cost functional is now taken to be a continuous functional over a suitable class of continuous functions. We also discuss an application of these results to control systems with stochastic boundary conditions.
\end{abstract}

\section{Introduction}

In the present paper, we prove an existence theorem for control systems whose state variables for every $t$ are in $C$, the set of continuous functions varying over a given set $I$. The dependence of the state variable upon $a \in I$ is induced by its dependence upon the initial state and the state equation governing the system. In contrast, the admissible controls $u=u(t)$ are taken as measurable functions of $t$ alone. The usual space constraints and boundary conditions are also allowed to vary with $a \in I$, and the cost functional is now taken to be a continuous functional over a suitable class of continuous functions. We further assume that the initial time $T_{1}$ is fixed and allow the final time $t_{2}(a)$ to vary with $a \in I$. Thus, the cost functional is of the form $I=K\left[t_{2}(a)\right.$,

\footnotetext{
'Paper received August 26, 1969. This research was accomplished under Grant No. AFAFOSR-942-65. The author is grateful to Dr. Lamberto Cesari for his suggestions and assistance in the preparation of this paper.

${ }^{2}$ Assistant Professor, Department of Industrial Engineering, University of Michigan, Ann Arbor, Michigan.
} 
$\left.x\left(t_{2}(a), a\right)\right], a \in I$. In particular, our existence theorem applies to control systems with stochastic boundary conditions; for these, we can take as cost functional the expectation $I=\int_{I} g\left[t_{2}(a), x\left(t_{2}(a), a\right)\right] d P$, where $g$ is a continuous, real-valued function and $P$ is a probability measure over $I$.

The central result of this paper is the existence theorem of Section 4 for control problems with state variable in $C$. In turn, this theorem is based on a closure theorem with state variable in $C$ which is new and is proved by the use of a new technique along with the general approach of Cesari for the case of state variables in $E_{n}$.

In Section 2, we formally present our control systems. In Section 3, we prove various properties of the systems; and, in Section 4, using these properties, we prove the closure theorem and the existence theorem. Finally, in Section 5, we discuss an application of our results to control systems with stochastic boundary conditions. In a future paper, we will present necessary conditions for such systems, these conditions being analogous to Pontryagin's necessary conditions for usual control systems.

\section{Description of the System}

We consider control systems whose initial conditions are given in terms of a known continuous function and whose state equations may also contain a known continuous function. In contrast, the control is required to be a function of time alone. More precisely, assume $I \subset E_{l}, I$ compact, and $i(a)$ a known continuous function from $I$ into $E_{n}$. Let $A(a), a \in I$, denote the constraint sets, where $A(a)$ are compact subsets of the $t x$-space $E_{1} \times E_{n}$, with $t$ in $E_{1}$, and $x=\left(x_{1}, \ldots, x_{n}\right)$, the space variable, in $E_{n}$. We assume that $A=\bigcup_{a \in I} A(a)$ is compact. Let $T_{1}<T_{2}$ be fixed times. For each $t$ in $\left[T_{1}, T_{2}\right]$, let $U(t)$, the control set, be a subset of the $u$-space $E_{m}$, $u=\left(u_{1}, \ldots, u_{m}\right)$, the control variable. Let $M(a)=\{(t, x, u):(t, x) \in A(a)$, $u(t) \in U(t)\}$ and $M=\bigcup_{a \in I} M(a)=\{(t, x, u):(t, x) \in A, u \in U(t)\}$ be compact subsets of $E_{1+n+m}$, and let $f=\left(f_{1}, \ldots, f_{n}\right)$ be a continuous vector function from $M$ into $E_{n}$ (see also Remark 2.2). Let $B(a)$, the terminal sets, $a \in I$, be closed subsets of points of $E_{1+n}$ and let $B=\bigcup_{a \in I} B(a)$. Let $T_{1}$ denote the fixed initial time, and let $\tau$, the set of terminal times $t_{2}(a), a \in I$, be a given family of equicontinuous functions, $T_{1} \leqslant t_{2}(a) \leqslant T_{2}$, which is closed in the uniform topology (see Remark 2.1). In order to guarantee that, for each fixed $t$ in $\left[T_{1}, T_{2}\right]$, the state $x(t, a), a \in I$, is a member of $C$, the set of continuous functions over $I$, we assume that there is a finite constant $d$ such that

$$
\left|f\left(t, x^{1}, u\right)-f\left(t, x^{2}, u\right)\right| \leqslant d\left|x^{1}-x^{2}\right|
$$


for all $\left(t, x^{1}, u\right),\left(t, x^{2}, u\right)$ in $M$, that is, $f$ is Lipschitzian in $x$ uniformly in $t$ and $u$ over the set $M$.

We shall now consider the class $\Omega$ of all pairs $x(t, a), u(t), T_{1} \leqslant t \leqslant T_{2}$, $T_{1} \leqslant t_{2}(a) \leqslant T_{2}, a \in I$, of the family of vector functions $x(t, a)$ and the vector function $u(t)$, satisfying the following conditions: (a) $x(t, a)$ is absolutely continuous (AC) in $\left[T_{1}, T_{2}\right]$ for each fixed $a \in I$; (b) $u(t)$ is measurable in $\left[T_{1}, T_{2}\right]$ (a function of $t$ alone); (c) $(t, x(t, a)) \in A(a), T_{1} \leqslant t \leqslant T_{2}$, for each $a \in I ; \quad$ (d) $u(t) \in U(t), T_{1} \leqslant t \leqslant T_{2} ; \quad$ (e) $x\left(T_{1}, a\right)=i(a) ; \quad$ (f) $t_{2}(\cdot) \in \tau$; (g) $\left(t_{2}(a), x\left(t_{2}(a), a\right)\right) \in B(a)$ for each $a \in I$; and (h) the state equation

$$
d x(t, a) / d t=f(t, x(t, a), u(t))
$$

is satisfied a.e. in $\left[T_{1}, T_{2}\right]$ for each $a \in I$ (see also Remark 2.2).

A pair $x(t, a), u(t), \quad T_{1} \leqslant t \leqslant T_{2}, T_{1} \leqslant t_{2}(a) \leqslant T_{2}, a \in I$, satisfying conditions (a)-(h) is said to be an admissible pair, where $x(t, a)$ is called a trajectory and $u(t)$ is called a control.

Let $K\left[w_{1}(\cdot), w_{2}(\cdot)\right]$ be a continuous functional, in the uniform topology, defined on the set of functions $W=\left\{\left(w_{1}(a), w_{2}(a)\right), \quad a \in I,: w_{i} \in C, \quad\left(t_{2}(a)\right.\right.$, $\left.w_{2}(a)\right) \in B(a)$ for each $\left.a \in I, i=1,2\right\}$. Assume that $K$ is bounded from below on $W^{\prime}=\left\{\left(w_{1}(a), w_{2}(a)\right), w_{i} \in C,\left(t_{2}(a), w_{i}(a)\right) \in B(a) \cap A(a), i=1,2\right\} \subset W$. Then, the functional

$$
\begin{aligned}
I[x, u] & =K[\eta(x)(a)], & & a \in I \\
& =K\left[t_{2}(a), x\left(t_{2}(a), a\right)\right], & & a \in I
\end{aligned}
$$

is called the cost functional, where $\eta(x)(a)=\left(t_{2}(a), x\left(t_{2}(a), a\right)\right), a \in I$.

We seek the absolute minimum of $I[x(\cdot, \cdot), u(\cdot)]$ in the class $\Omega$. If $(\tilde{x}(\cdot, \cdot), \tilde{u}(\cdot))$ has the property that $I[\tilde{x}(\cdot, \cdot), \tilde{u}(\cdot)] \leqslant I[x(\cdot, \cdot), u(\cdot)]$ for all $(x(\cdot, \cdot), u(\cdot)) \in \Omega$, then we say that $\tilde{x}(\cdot, \cdot), \tilde{u}(\cdot)$ is an optimal pair; and we may say that $\tilde{u}(\cdot)$ is an optimal control and $\tilde{x}(\cdot, \cdot)$ is an optimal trajectory. Though the optimal pair $\tilde{x}(\cdot, \cdot), \tilde{u}(\cdot)$ may not be unique in $\Omega$, the value of the cost functional $I[\tilde{x}(\cdot, \cdot), \tilde{u}(\cdot)]$ is the same for all optimal pairs.

Remark 2.1. In the description of $\Omega$, the class of admissible pairs, we require, for various technical reasons, that there is a finite time $T_{2}$ such that $T_{1} \leqslant t_{2}(a) \leqslant T_{2}$ for all $a \in I$ and such that the solution to the state equation exists over all of $\left[T_{1}, T_{2}\right]$. Physically, we may interpret this as demanding that a safety margin exists, that is, we ask that, if we ignore our boundary conditions, we can extend our trajectories past the prescribed stopping time $t_{2}(a)$ to time $T_{2}$. Moreover, in condition (f), for the purpose of obtaining a minimizing sequence, we also require that $t_{2}(a), a \in I$, belongs to an equicontinuous 
family $\tau$ which is closed in the uniform topology. For example, we may require that $t_{2}(a)$ be constant for $a \in I$ or we may take $\tau$ to be the class of all Lipschitz functions over $I$ with a fixed Lipschitz constant. We can think of this requirement as arising from the nature of the instruments governing the stopping time.

Remark 2.2. The control systems described in this section also include systems in which the state function $f$ contains the parameter $a$, that is, where Eq. (1) is replaced by

$$
d x(t, a) / d t=f(t, x(t, a), u(t), a)
$$

and where $f$ is a continuous vector function from $M \times I$ into $E_{n}$. Indeed, in this case, we have only to consider the additional variables $z=\left(x_{n+1}, \ldots\right.$, $\left.x_{n+l}\right)$ satisfying $d x_{i} / d t=0, x_{i}\left(T_{1}, a\right)=a, i=n+1, \ldots, n+l$. Then, if $\tilde{x}=\left(x_{1}, \ldots, x_{n}, x_{n+1}, \ldots, x_{n+l}\right)=(x, z)$ and $f_{i}=0, i=n+1, \ldots, n+l$, we have the system

$$
d \tilde{x}(t, a) / d t=\tilde{f}(t, \tilde{x}(t, a, b), u(t))
$$

with $f_{i}(t, \tilde{x}, u)=f_{i}(t, x, z, u), i=1, \ldots, n+l$, which is of the form we first presented. More generally, the control systems described in this section also include systems in which the state function $f$ contains a perturbation $\eta(t, b) \in E_{s}$, that is, whose state equation is given by

$$
d x(t, a, b) / d t=f(t, x(t, a, b), u(t), \eta(t, b))
$$

where $\eta(\cdot, \cdot)$ satisfies conditions similar to those of $x$. In particular, assume that (i) the parameter $b$ varies over some compact set $I_{0}$ in Euclidean space $E_{p}$, (ii) for each fixed $b \in I_{0}, \eta^{\prime}(t, b)$ exists a.e. in $\left[T_{1}, T_{2}\right]$ and satisfies $\eta^{\prime}(t, b)=\gamma(t, \eta, b)$ with $(t, \eta) \in A_{0} \subset E_{1+s}$ compact, (iii) $\eta\left(T_{1}, b\right)=j(b), j$ a continuous function on $I_{0}$, and (iv) $\gamma$ is Lipschitzian in $\eta$ and $b$, uniformly in $t$, over the set $A_{0} \times I_{0}$. Such systems can now be rewritten in the previous form by introducing the variable $z \in E_{p}$ and setting $\tilde{x}=(x, \eta, z)$, where $z(t, b)=b$. We then obtain the system

$$
\begin{aligned}
d \tilde{x}(t, a, b) / d t & =(f(t, x(t, a, b), \eta(t, b), u(t)), \gamma(t, \eta(t, b), z(t, b)), 0) \\
& =\tilde{f}(t, \tilde{x}(t, a, b), u(t))
\end{aligned}
$$

with initial conditions $\tilde{x}\left(T_{1}, a, b\right)=(i(a), j(b), b)$ and, hence, we are back to our previous form. 


\section{Properties of Equicontinuity of the State Variable}

The following properties of the systems just described will be used in the next section.

Property 3.1. Consider a control system as described in Section 2. Then, $x(t, a)$ is uniformly continuous for $(t, a) \in\left[T_{1}, T_{2}\right] \times I$. Moreover, given a sequence $\left[x_{k}(\cdot, \cdot), u_{k}(\cdot)\right]$ of admissible pairs, then $x_{k}(\cdot, \cdot), k=1,2, \ldots$, forms an equicontinuous and equibounded family of functions in $\left[T_{1}, T_{2}\right] \times I$.

Proof. Let $x(\cdot, \cdot), u(\cdot)$ be an admissible pair. By the Lipschitz assumption on $f$, if $a_{1}, a_{2} \in I$,

$$
\begin{aligned}
\left|x\left(t, a_{1}\right)-x\left(t, a_{2}\right)\right| & \leqslant\left|i\left(a_{1}\right)-i\left(a_{2}\right)\right|+\int_{T_{1}}^{t}\left|f\left(s, x\left(s, a_{1}\right), u(s)\right)-f\left(s, x\left(s, a_{2}\right), u(s)\right)\right| d s \\
& \leqslant\left|i\left(a_{1}\right)-i\left(a_{2}\right)\right|+d \int_{T_{1}}^{T_{2}}\left|x\left(s, a_{1}\right)-x\left(s, a_{2}\right)\right| d s, \quad T_{1} \leqslant t \leqslant T_{2}
\end{aligned}
$$

Hence, by Gronwall's inequality,

$$
\left|x\left(t, a_{1}\right)-x\left(t, a_{2}\right)\right| \leqslant C\left|i\left(a_{1}\right)-i\left(a_{2}\right)\right|
$$

$C=\exp \left[d\left(T_{2}-T_{1}\right)\right]<\infty, T_{1} \leqslant t \leqslant T_{2}$. Note that $C$ does not depend upon $u(\cdot)$. Since $f$ is continuous over the compact set $M$, there is a finite $K$, not dependent upon $u(\cdot)$, such that $|f|<K$ on $M$. Hence, by (2), for $\left(t_{1}, a_{1}\right)$, $\left(t_{2}, a_{2}\right)$ in $\left[T_{1}, T_{2}\right] \times I$,

$$
\begin{aligned}
\left|x\left(t_{1}, a_{1}\right)-x\left(t_{2}, a_{2}\right)\right| & \leqslant\left|x\left(t_{1}, a_{1}\right)-x\left(t_{2}, a_{1}\right)\right|+\left|x\left(t_{2}, a_{1}\right)-x\left(t_{2}, a_{2}\right)\right| \\
& \leqslant K\left|t_{1}-t_{2}\right|+C\left|i\left(a_{1}\right)-i\left(a_{2}\right)\right|
\end{aligned}
$$

Since $i$ ( $a$ ) is continuous for $a \in I, I$ compact, $i(a)$ is uniformly continuous over $I$. Thus, from (3), it follows that $x(t, a)$ is uniformly continuous over $\left[T_{1}, T_{2}\right] \times I$. Moreover, since $K$ and $C$ are independent of $u(\cdot)$, if $\left[x_{k}(\cdot, \cdot), u_{k}(\cdot)\right]$ is a sequence of admissible pairs, (3) holds with $x$ replaced by $x_{k}$, that is,

$$
\left|x_{k}\left(t_{1}, a_{1}\right)-x_{k}\left(t_{2}, a_{2}\right)\right| \leqslant K\left|t_{1}-t_{2}\right|+C\left|i\left(a_{1}\right)-i\left(a_{2}\right)\right|, \quad k=1,2, \ldots
$$

Hence, $x_{k}(t, a),(t, a) \in\left[T_{1}, T_{2}\right] \times I, k=1,2, \ldots$, forms an equicontinuous family of functions over $\left[T_{1}, T_{2}\right] \times I$. Since $A$ is assumed compact, this family is also equibounded. Property 3.1 is thereby proved. 
Property 3.2. Consider a sequence $\left[x_{n}(t, a), T_{1} \leqslant t \leqslant T_{2}, a \in I\right]$ of equibounded and equicontinuous functions of $(t, a)$ in $\left[T_{1}, T_{2}\right] \times I$. Moreover, suppose that, for each $n$, there is an associated function $t_{2 n}(a), a \in I$, $T_{1} \leqslant t_{2 n}(a) \leqslant T_{2}$, with $t_{2 n}(\cdot) \in \tau, n=1,2, \ldots$. Then, there exists at least one subsequence $\left[x_{n s}(\cdot, \cdot)\right]$ of $\left[x_{n}(\cdot, \cdot)\right]$ which converges uniformly in $t$ and $a$ to a continuous function $x(t, a), T_{1} \leqslant t \leqslant T_{2}, a \in I$, and for which $t_{2 n s}(a)$ converges uniformly in $a$ to a continuous function $t_{2}(a), a \in I$, with $t_{2}(\cdot) \in \tau$ (see Ref. 1, Chapter 7).

\section{Closure and Existence Theorem}

We now impose further requirements on the state equation and the control set $U(t)$ and prove a closure theorem and an existence theorem for the resulting systems. The central result of this section is the closure theorem. The main step in its proof is the construction of a new control system with the state variable in $E_{2 n}$, this system being obtained from the general system by fixing two points $a_{1}$ and $a_{2}$ in $I$. We then use the results of Cesari (Ref. 2) and the form of the state function $f$ given below in assumption $(\alpha)$ to complete the proof. In particular, let us assume that $(\alpha) f(t, x, u)=g(t, x)+k(t, u)$ for $(t, x, u)$ in $M$. We shall also require, as usual, the following assumptions: $(\beta) U(t)$ is a compact subset of $E_{m}$ for $t$ in $\left[T_{1}, T_{2}\right] ;(\gamma) U(t)$ is an upper semicontinuous function of $t$ in $\left[T_{1}, T_{2}\right]$; and $(\delta) k(t, U(t))=\left\{y \in E_{n}: y=\right.$ $k(t, u), u \in U(t)\}$ is a convex subset of $E_{n}$ for each $t$ in $\left[T_{1}, T_{2}\right]$; consequently, $Q(t, x)=f(t, x, U(t))$ is a convex subset of $E_{n}$ for each $(t, x) \in A$.

Closure Theorem. Let $A(a), a \in I$, and $A$ be compact subsets of $E_{1+n}$, let $U(t)$ be a compact subset of $E_{m}$ for every $t$ in $\left[T_{1}, T_{2}\right]$, let $U(t)$ be an upper semicontinuous function of $t$ in $\left[T_{1}, T_{2}\right]$, let $f(t, x, u)=g(t, x)+k(t, u)$ be a continuous function on $M$ into $E_{n}$, let $M(a), a \in I$, and $M$ be compact subsets of $E_{1+n+m}$, and let $k(t, U(t))$ be a convex subset of $E_{m}$ for every $(t, x) \in A$. Let $x_{k}(t, a), T_{1} \leqslant t \leqslant T_{2}, a \in I$, with $\left(t_{2 k}(a), a \in I\right) \in \tau$, be a sequence of trajectories satisfying assumptions (a)-(f) and (h) of Section 2 and converging uniformly toward a function $x_{0}(t, a), T_{1} \leqslant t \leqslant T_{2}, a \in I$, which is AC in $\left[T_{1}, T_{2}\right]$ for each $a \in I$. Also, suppose that $t_{2 h}(\cdot)$ converges uniformly to $t_{2}(\cdot)$. Then, there exists a measurable $u_{0}(\cdot), u_{0}(t) \in U(t), T_{1} \leqslant t \leqslant T_{2}$, such that the pair $x_{0}(t, a), u_{0}(t), T_{1} \leqslant t \leqslant T_{2}, a \in I$, with $t_{2}(a), a \in I$, satisfies conditions (a)-(f) and (h) of Section 2 ; that is, with the exception of condition $(g), x_{0}(\cdot, \cdot)$ is an admissible trajectory with stopping time $t_{2}(\cdot) \in \tau$, and $\eta\left[x_{k}(\cdot, \cdot)\right](a)$ converges uniformly to $\eta\left[x_{0}(\cdot, \cdot)\right](a), a \in I$. 
Proof. For each $a \in I$, the vector functions $x_{0}{ }^{\prime}(t, a), x_{k}{ }^{\prime}(t, a)=$ $f\left(t, x_{k}(t, a), u_{k}(t, a)\right), T_{1} \leqslant t \leqslant T_{2}, k=1,2, \ldots$, are defined a.e. and are $L$-integrable. We have to prove that, for each $a \in I,\left(t, x_{0}(t, a)\right) \in A(a)$, $T_{1} \leqslant t \leqslant T_{2}$, that $t_{2}(\cdot) \in \tau$, and that there is a measurable function $u_{0}(t)$, $T_{1} \leqslant t \leqslant T_{2}$, such that, for each $a \in I$,

$$
x_{0}^{\prime}(t, a)=f\left(t, x_{0}(t, a), u_{0}(t)\right), u_{0}(t) \in U(t) \quad \text { a.e. in } \quad\left[T_{1}, T_{2}\right]
$$

First, from $x_{k}(\cdot, \cdot) \rightrightarrows x_{0}(\cdot, \cdot)$ (where $\rightarrow$ denotes uniform convergence) and the assumption that $\left(t, x_{k}(t, a)\right) \in A(a),(t, a) \in\left[T_{1}, T_{2}\right] \times I, A(a)$ closed, it follows that $\left(t, x_{0}(t, a)\right) \in A(a),(t, a) \in\left[T_{1}, T_{2}\right] \times I$. It is immediate from condition (f) of Section 2 that $t_{2}(\cdot) \in \tau$. Since $f$ is continuous on the compact set $M$, there exists a finite $K$ such that $|f| \leqslant K$ over $M$. Hence, for each $a \in I$,

$$
\left|x_{k}\left(t^{\prime}, a\right)-x_{k}\left(t^{\prime \prime}, a\right)\right| \leqslant K\left|t^{\prime}-t^{\prime \prime}\right|
$$

for any $t^{\prime}, t^{\prime \prime}$ in $\left[T_{1}, T_{2}\right]$. Thus, for $a \in I$,

$$
\begin{aligned}
\left|x_{k}\left(t_{2 k}(a), a\right)-x_{0}\left(t_{2}(a), a\right)\right| & \leqslant\left|x_{k k}\left(t_{2 k}(a), a\right)-x_{k}\left(t_{2}(a), a\right)\right|+\left|x_{k i}\left(t_{2}(a), a\right)-x_{0}\left(t_{2}(a), a\right)\right| \\
& \leqslant K\left|t_{2 k}(a)-t_{2}(a)\right|+\left|x_{k}\left(t_{2}(a), a\right)-x_{0}\left(t_{2}(a), a\right)\right|
\end{aligned}
$$

from which it follows that $x_{k}\left(t_{2 k}(a), a\right) \rightrightarrows x_{0}\left(t_{2}(a), a\right)$ and $\eta\left[x_{k}(\cdot, \cdot)\right](a) \rightrightarrows$ $\eta\left[x_{0}(\cdot, \cdot)\right](a)$. Hence, it only remains to find a measurable $u_{0}(t)$ satisfying (4).

It is immediate that, for each $a \in I, x_{k}(\cdot, a) \rightarrow x_{0}(\cdot, a), x_{0}(t, a) \mathrm{AC}$ in $\left[T_{1}, T_{2}\right]$. Moreover, for each $a \in I$, if we consider the control system with constraint set $A(a)$, control set $U(t)$, and state equation $x^{\prime}=f(t, x, u)$, with $T_{1} \leqslant t \leqslant T_{2}$, then each of the pairs $x_{k}(\cdot, a), u_{k}(\cdot), k=1,2, \ldots$, belongs to $\Omega(a)$, the class of admissible pairs for this system. Hence, we can conclude from Cesari's closure theorem (see Ref. 2) that, for each $a \in I$, there exists a measurable $u_{0}(\cdot, a), u_{0}(t, a) \in U(t), T_{1} \leqslant t \leqslant T_{2}$, such that

$$
x_{0}{ }^{\prime}(t, a)=f\left(t, x_{0}(t, a), u_{0}(t, a)\right)
$$

a.e. in $\left[T_{1}, T_{2}\right]$ and, in particular, $\left(x_{0}(\cdot, a), u_{0}(\cdot, a)\right) \in \Omega(a)$. We will show that, for any fixed $a_{0} \in I, u_{0}\left(\cdot, a_{0}\right)$ generates all the trajectories $x_{0}(\cdot, a), a \in I$; that is, for any $a \in I$,

$$
x_{0}{ }^{\prime}(t, a)=f\left(t, x_{0}(t, a), u_{0}\left(t, a_{0}\right)\right)
$$

a.e. in $\left[T_{1}, T_{2}\right]$. If one sets $u_{0}(t)=u_{0}\left(t, a_{0}\right)$, this will then complete the proof.

We now show that, for any $a_{1}, a_{2} \in I, u_{0}\left(\cdot, a_{1}\right)$ generates $x_{0}\left(\cdot, a_{2}\right)$. To this end, let us consider the control system consisting of the constraint set 
$\widetilde{A}=\left\{(t, X):\left(t, X, \ldots, X_{n}\right) \in A\left(a_{1}\right),\left(t, X_{n+1}, \ldots, X_{2 n}\right) \in A\left(a_{2}\right)\right\}$, a compact subset of the $t X$-space $E_{1} \times E_{2 n}$, with $t$ in $E_{1}$ and $X=\left(X_{1}, \ldots, X_{n}, \ldots, X_{2 n}\right)$, the state variable, in $E_{2 n}$. Let $T_{1}, T_{2}$ be given as before; and, for each $t$ in $\left[T_{1}, T_{2}\right]$, let the control set $U(t)$ be the same as before, with $u=\left(u_{1}, \ldots, u_{m}\right)$ the control variable. Hence, if $\tilde{M}=\{(t, X, u):(t, X) \in \tilde{A}, u \in U(t)\}$, then $\tilde{M}=\left\{(t, X, u):\left(t, X_{1}, \ldots, X_{n}, u\right) \in M\left(a_{1}\right),\left(t, X_{n+1}, \ldots, X_{2 n}, u\right) \in M\left(a_{2}\right)\right\}$ is a compact subset of $E_{1+2 n+m}$. Let $F=\left(F_{1}, \ldots, F_{2 n}\right)$ be a continuous vector function from $\tilde{M}$ into $E_{2 n}, F(t, X, u)$ given by

$$
F(t, X, u)=\left(f\left(t, X_{1}, \ldots, X_{n}, u\right), f\left(t, X_{n+1}, \ldots, X_{2 n}, u\right)\right)
$$

The class $\widetilde{\Omega}$ of admissible pairs $X(t), u(t), T_{1} \leqslant t \leqslant T_{2}$, consists of those pairs satisfying the following conditions: $(\mathrm{A}) X(t)$ is $\mathrm{AC}$ in $\left[T_{1}, T_{2}\right] ;(\mathrm{B}) u(t)$ is measurable in $\left[T_{1}, T_{2}\right]$; (C) $(t, X(t)) \in \tilde{A}$ for $T_{1} \leqslant t \leqslant T_{2}$; (D) $u(t) \in U(t)$ for $T_{1} \leqslant t \leqslant T_{2} ;(\mathrm{E}) X\left(t_{1}\right)=\left(i\left(a_{1}\right), i\left(a_{2}\right)\right)$; and (F) the state equation

$$
d X(t) / d t=F(t, X(t), u(t))
$$

is satisfied a.e. in $\left[T_{1}, T_{2}\right]$.

By the construction of this control system, it is clear that $\left(X_{k}(\cdot), u_{k}(\cdot)\right)=$ $\left(\left(x_{k}\left(\cdot, a_{1}\right), x_{k}\left(\cdot, a_{2}\right)\right), u_{k}(\cdot)\right) \in \widetilde{\Omega}, k=1,2, \ldots$. We wish to show that $X_{0}(t)=$ $\left(x_{0}\left(t, a_{1}\right), x_{0}\left(t, a_{2}\right)\right), T_{1} \leqslant t \leqslant T_{2}$, is an admissible trajectory for this system. Again, it is immediate that $X_{k}(t) \rightarrow X_{0}(t), T_{1} \leqslant t \leqslant T_{2}, X_{0}(t) \mathrm{AC}$ in $\left[T_{1}, T_{2}\right]$. Moreover, since $A\left(a_{i}\right)$ and $M\left(a_{i}\right)$ were assumed compact, $i=1,2, \tilde{A}$ and $\tilde{M}$ are compact. In addition, $\widetilde{Q}(t, X)=F(t, X, U(t))$ is convex by assumption $(\alpha)$ since, for fixed $(t, X) \in \widetilde{A}$ and any $u_{1}, u_{2}$ in $U(t)$, we have, for $0 \leqslant \alpha \leqslant 1$,

$$
\begin{aligned}
\alpha(f(t, & \left.\left.X_{1}, \ldots, X_{n}, u_{1}\right), f\left(t, X_{n+1}, \ldots, X_{2 n}, u_{1}\right)\right) \\
& \quad+(1-\alpha)\left(f\left(t, X_{1}, \ldots, X_{n}, u_{2}\right), f\left(t, X_{n+1}, \ldots, X_{2 n}, u_{2}\right)\right) \\
= & \left(g\left(t, X_{1}, \ldots, X_{n}\right)+\alpha k\left(t, u_{1}\right)+(1-\alpha) k\left(t, u_{2}\right), g\left(t, X_{n+1}, \ldots, X_{2 n}\right)\right. \\
& \left.\quad+\alpha k\left(t, u_{1}\right)+(1-\alpha) k\left(t, u_{2}\right)\right) \\
= & \left(g\left(t, X_{1}, \ldots, X_{n}\right)+k\left(t, u_{\alpha}\right), g\left(t, X_{n+1}, \ldots, X_{2 n}\right)+k\left(t, u_{\alpha}\right)\right) \\
= & \left(f\left(t, X_{1}, \ldots, X_{n}, u_{\alpha}\right), f\left(t, X_{n+1}, \ldots, X_{2 n}, u_{\alpha}\right)\right)
\end{aligned}
$$

where, by assumption $(\delta)$, there exists a $u_{\alpha} \in U(t)$ such that

$$
k\left(t, u_{\alpha}\right)=\alpha k\left(t, u_{1}\right)+(1-\alpha) k\left(t, u_{2}\right)
$$

Hence, we can apply Cesari's closure theorems (Ref. 2) to conclude that there exists a measurable control function $u_{0}(t), T_{1} \leqslant t \leqslant T_{2}$, such that

$$
X_{0}^{\prime}(t)=F\left(t, X_{0}(t), u_{0}(t)\right), \quad u_{0}(t) \in U(t)
$$


a.e. in $\left[T_{1}, T_{2}\right]$. By the construction of $X_{0}(t), F(t, x, u)$, and (6), it thus follows that

$$
\begin{aligned}
& f\left(t, x_{0}\left(t, a_{1}\right), u_{0}\left(t, a_{1}\right)\right)=f\left(t, x_{0}\left(t, a_{1}\right), u_{0}(t)\right) \\
& f\left(t, x_{0}\left(t, a_{2}\right), u_{0}\left(t, a_{2}\right)\right)=f\left(t, x_{0}\left(t, a_{2}\right), u_{0}(t)\right)
\end{aligned}
$$

a.e. in $\left[T_{1}, T_{2}\right]$. Hence, $k\left(t, u_{0}\left(t, a_{1}\right)\right)=k\left(t, u_{0}\left(t, a_{2}\right)\right)$ a.e. in $\left[T_{1}, T_{2}\right]$ and, therefore, $f\left(t, x_{0}\left(t, a_{2}\right), u_{0}\left(t, a_{2}\right)\right)=f\left(t, x_{0}\left(t, a_{2}\right), u_{0}\left(t, a_{1}\right)\right)$ a.e. in $\left[T_{1}, T_{2}\right]$, that is, $u_{0}\left(t, a_{1}\right)$ generates $x_{0}\left(t, a_{2}\right), T_{1} \leqslant t \leqslant T_{2}$. Since $a_{1}$ and $a_{2}$ were chosen arbitarily from $I$, we have thereby verified relation (7); and, by previous remarks, the closure theorem is thereby proved.

Existence Theorem. Consider a control system as described in Section 2 and satisfying conditions $(\alpha)-(\delta)$. Assume that the class of admissible pairs $\Omega$ is nonempty. Then, there exists an admissible pair $x_{0}(\cdot, \cdot), u_{0}(\cdot)$, such that $I\left[x_{0}(\cdot, \cdot), u_{0}(\cdot)\right] \leqslant I[x(\cdot, \cdot), u(\cdot)]$ for all admissible pairs $x(\cdot, \cdot), u(\cdot)$.

Proof. By assumption, if $(x(\cdot, \cdot), u(\cdot)) \in \Omega$, then $\eta[x(\cdot, \cdot)](\cdot) \in W^{\prime}$. Since $K[\eta[x(\cdot, \cdot)](a)], a \in I$, is assumed to be bounded from below on $W^{\prime}$, $i=\inf _{\Omega} I[x(\cdot, \cdot), u(\cdot)]>-\infty$. Since there exists at least one admissible pair by assumption, $i$ is therefore finite. Thus, there exists a minimizing sequence of admissible pairs $x_{k}(t, a), u_{k}(t), T_{1} \leqslant t \leqslant T_{2}, a \in I$, with $t_{2 k}(\cdot) \in \tau$, such that $I\left[x_{k}(\cdot, \cdot), u_{k}(\cdot)\right] \rightarrow i$ as $k \rightarrow \infty$.

By Property $3.1, x_{k}(\cdot, \cdot)$ forms an equicontinuous and equibounded family of functions in $\left[T_{1}, T_{2}\right] \times I, k=1,2, \ldots$. By Property 3.2 , there exists a subsequence, which we still call $\left[x_{k}(\cdot, \cdot)\right]$, which converges uniformly to a continuous function $x_{0}(t, a),(t, a) \in\left[T_{1}, T_{2}\right] \times I$ and for which $t_{2 h}(a)$ converges uniformly to a continuous function $t_{2}(a) \in \tau, a \in I$. Moreover, from (5), it follows that, for any $a \in I,\left|x_{0}\left(t^{\prime}, a\right)-x_{0}\left(t^{\prime \prime}, a\right)\right| \leqslant K$ for any $t^{\prime}, t^{\prime \prime}$ in $\left[T_{1}, T_{2}\right]$, so that $x_{0}(\cdot, a)$ is Lipschitzian, and hence $A C$, in $\left[T_{1}, T_{2}\right]$, for any $a \in I$. Consequently, by the closure theorem, there exists a measurable $u_{0}(t), u_{0}(t) \in U(t), T_{1} \leqslant t \leqslant T_{2}$, such that the pair $x_{0}(\cdot, \cdot), u_{0}(\cdot)$ satisfies assumptions (a)-(f) and (h) of Section 2 and such that $\eta\left[x_{k}(\cdot, \cdot)\right](a)$ converges uniformly to $\eta\left[x_{0}(\cdot, \cdot)\right](a), a \in I$. Since $B(a)$ is assumed closed for each $a \in I$ by assumption, it follows that $\eta\left[x_{0}(\cdot, \cdot)\right](a)=\left(t_{2}(a), x_{0}\left(t_{2}(a), a\right) \in B(a)\right)$ for each $a \in I$; that is, assumption (g) of Section 2 is also satisfied. Thus, $\left(x_{0}(\cdot, \cdot), u_{0}(\cdot)\right) \in \Omega$. Finally, since $K$ is assumed continuous in $W$,

$$
I\left[x_{0}(\cdot, \cdot), u_{0}(\cdot)\right]=\lim _{k \rightarrow \infty} I\left[x_{k}(\cdot, \cdot), u_{k}(\cdot)\right]=i .
$$

The existence theorem is thereby proved. 


\section{Stochastic Systems}

Let us now consider a class of control systems with stochastic boundary conditions. Specifically, let us particularize the control systems considered thus far by making $i(a), a \in I$, a random variable, and by writing the cost functional as an expectation. In particular, let us assume that we are given a probability measure $P$ over the Borel sets restricted to $I$. Again, we consider a control system as described in Section 2, with the cost functional now given as follows.

Assume that $h(t, y)$ is a real-valued, continuous function on the set $B \subset E_{1+n}$. Then, the cost functional is given by

$$
I[x(\cdot, \cdot), u(\cdot)]=E h\left(t_{2}(a), x\left(t_{2}(a), a\right)\right)=\int_{I} h\left(t_{2}(a), x\left(t_{2}(a), a\right)\right) d P
$$

where the letter $E$ denotes the expectation with respect to the probability measure $P$. We also assume that the set $B$ is closed in $E_{1+n}$. Thus, $h(\cdot, \cdot)$ is continuous, and hence bounded, on the compact set $B \cap A$; and it is then immediate that this cost functional has all the required properties of the functional $K$. Therefore, these systems are subsumed by those described in Section 2. In other words, the state equation is a differential equation with stochastic initial conditions $i(a)$, with probability distribution $P$ on the space $I$ of the variable $a$. Hence, for a given control $u(\cdot)$, we obtain, for each $t$, a family $x(t, a)$ of vector-valued functions of $a$ in $I$. Again, the probability measure $P$ and the function $h$ are also used to describe explicitly the cost functional $K$. Consequently, the results of Section 4 are applicable to these stochastic systems.

Such systems can arise, for example, if the interval $\left[T_{1}, T_{2}\right]$ is thought of as an interval of ignorance for a usual control system, during which the initial conditions and the state of the system are unknown. Alternately, such a system can arise if we are forced to choose a control $u(\cdot)$ before the initial conditions are known. For a further discussion of such systems, see also Ref. 3, Chapter 2.

Remark 5.1. As shown in Remark 2.2, if we consider control systems whose state equation is of the form $x^{\prime}=g(t, x, \eta)+k(t, u), \eta$ a random variable or a stochastic process whose sample paths possess the same differentiability properties as $x(t, a)$, then clearly we can transform such systems into systems with stochastic initial conditions; hence, the results of Section 4 pertain to such systems as well (see Example 5.3; see also Ref. 3, Section 8.6). 
Example 5.1. Let $u$ consider the control system $\dot{x}_{1}=x_{2}, \dot{x}_{2}=u$, $x_{1}(0, a)=0, x_{2}(0, a)=a$, where $u(t) \in U=[-1 \leqslant u \leqslant 1]$ and $a$ is any real number between 0 and 1 , with probability density one. We wish to find admissible pairs which minimize $I[x(\cdot, \cdot), u(\cdot)]$ within $\Omega, I[x(\cdot, \cdot), u(\cdot)]=$ $E\left[x_{2}(T, a)\right]^{2}=\int_{0}^{1}\left[x_{2}(T, a)\right]^{2} d a$, with $0 \leqslant t \leqslant T, T$ fixed. If we further require that $(t, x(t, a))$ lie in some compact set $A$, then clearly this control system satisfies all the relevant conditions; hence, by the results of Section 4 , an optimal pair exists. Since $x_{2}(t, a)=a+\int_{0}^{t} u(s) d s$, it can be easily shown that, if $A$ is large enough to contain the trajectories described below, the optimal pairs can be described as follows:

Case 1: $T<\frac{1}{2}$. Set $u(t)=-1$ on $[0, T]$. Hence, $x_{2}(T, a)=a-T$.

Case 2: $\quad T \geqslant \frac{1}{2}$. Any admissible control such that $E x(T, a)=0$ and such that the space constraints are satisfied is optimal. For example, we may set $u(t)=-1$ on $\left[0, \frac{1}{2}\right]$ and $u(t)=0$ on $\left(\frac{1}{2}, T\right]$.

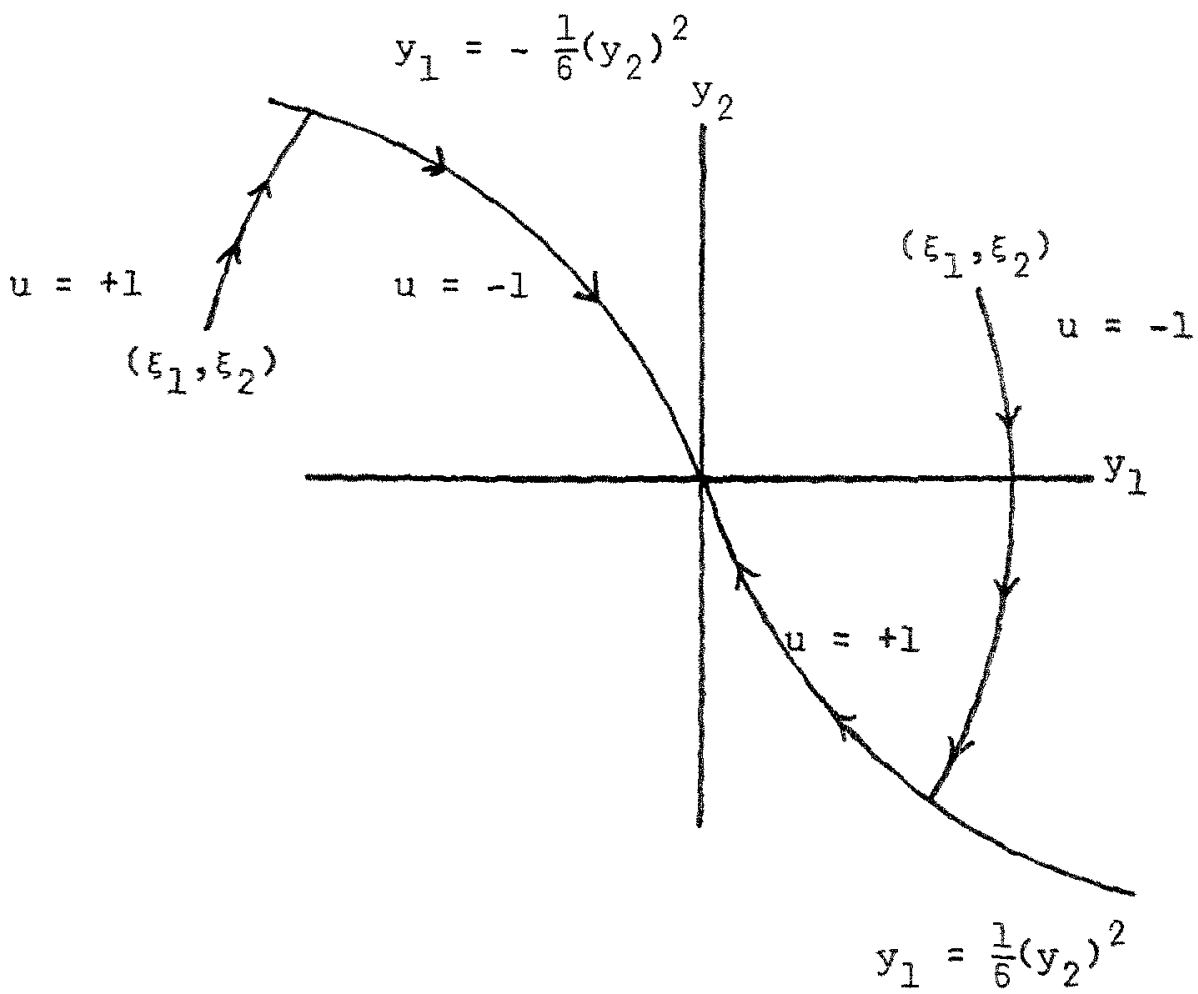

Fig. 1 
Example 5.2. Let us consider the control system of Example 5.1, with the cost functional now given by $E g\left(x_{2}(T, a)\right)$, where $g(x)=2 x, x \geqslant 0, g(x)=-4 x$, $x<0$. Again, if the constraint set is large enough, the results of Section 4 apply and the optimal pairs can be described as follows:

Case 1: $\quad T<\frac{1}{3}$. Set $u(t)=-1$ on $[0, T]$.

Case 2: $\quad T \geqslant \frac{1}{3}$. Any admissible control such that $E x_{2}(T, a)=\frac{1}{6}$ is optimal.

Note that, unlike Example 5.1, the optimal solution cannot be obtained by replacing $x$ with $E x$ throughout the system.

Example 5.3. Let us consider the control system of Example 5.1, with the state equation now given by $\dot{x}_{1}=a x_{2}, \dot{x}_{2}=u$, with fixed initial conditions $\left(\xi_{1}, \xi_{2}\right), 0 \leqslant t \leqslant T, T$ free, and where the cost functional is given by $E\left[\left(x_{1}(T, a)\right)^{2}+\left(x_{2}(T, a)\right)^{2}\right]$. From the above remarks, if the constraint set is large enough, the results of Section 4 are applicable. Moreover, the optimal pairs can be shown to be those admissible pairs with $\operatorname{Eax}_{1}(T, a)=0$ and $E x_{2}(T, a)=E x_{2}(T)=0$. If we set $y_{1}=E a x_{1}(t, a), y_{2}=E x_{2}(t, a)$, then $\dot{y}_{1}=\frac{1}{3} y_{2}, \dot{y}_{2}=u$, with $y_{1}(0)=\frac{1}{2} \xi_{1}, y_{2}(0)=\xi_{2}$. In particular, one set of optimal trajectories is described in Fig. 1.

\section{References}

1. Kellex, J. L., General Topology, D. Van Nostrand Company, Princeton, New Jersey, 1955.

2. Cesari, L., Existence Theorems for Weak and Usual Optimal Solutions in Lagrange Problems with Unilateral Constraints, $I$, Transactions of the American Mathematical Society, Vol. 124, No. 3, 1966.

3. BAUm, R., Optimal Control Systems with Stochastic Boundary Conditions, University of Michigan, Ph.D. Thesis, 1969.

\section{Additional Bibliography}

Fleminc, W. H., Optimal Continuous-Parameter Stochastic Control, SIAM Review, Vol. 11, No. 4, 1969.

KusHner, H. J., On the Existence of Optimal Stochastic Controls, SIAM Journal on Control, Vol. 3, No. 3, 1965. 